\title{
RECEPTIVIDAD Y RESISTENCIAS DEL DISCURSO DE LOS DERECHOS HUMANOS EN LOS ARBITRAJES DE INVERSIÓN
}

\author{
Receptivity and resistance of the human \\ rights in investment arbitrations
}

\author{
JENNER ALONSO TOBAR TORRES* \\ Universidad Libre de Colombia
}

\begin{abstract}
RESUMEN
El derecho internacional de inversiones ha cobrado cada vez mayor relevancia como herramienta de promoción y protección de las inversiones extranjeras, siendo el arbitraje de inversiones el principal escenario donde las controversias entre Estados receptores e inversionistas son resueltas. Sin embargo, en estos foros arbitrales los Derechos Humanos han observado una limitada receptividad, generando contextos de fragmentación entre aquellos y el derecho internacional de inversiones. El presente artículo ilustra la señalada tensión y mediante la identificación de algunas vías de acceso, defiende la tesis que las normas de Derechos Humanos pueden y deben ser tomadas en cuenta en la resolución de controversias arbitrales de inversión.
\end{abstract}

\section{PALABRAS CLAVE}

Derecho internacional de Inversiones, Derechos Humanos, Fragmentación del derecho internacional.

\section{ABSTRACT}

Abstract: International investment law has become increasingly important as a tool for the promotion and protection of foreign investments, with investment arbitration as the main scenario where disputes between host states and investors are resolved. However, in those arbitral forums, Human Rights have observed limited receptivity, generating fragmentation contexts with international investment law. This paper exposes the aforementioned tension and identifying some access routes, defends the thesis that Human Rights norms can and should be considered in the resolution of arbitration investment disputes.

\section{KEYWORDS}

International Investment Law, Human Rights, Fragmentation of international law.

\section{Introducción}

En las últimas décadas, el derecho internacional de inversiones ha registrado un exponencial crecimiento a partir de la notable proliferación de instrumentos internacionales dirigidos a crear marcos de protección a las inversiones que los particulares efectúan en suelo extranjero. En la actualidad se pueden identificar al menos tres instrumentos bajo los cuales los Estados negocian los estándares sustantivos de protección a la inversión extranjera, a saber, los tratados bilaterales de inversión (también denominados Acuerdos para la Promoción y Protección Recíproca de las Inversiones), los capítulos de protección a la inversión contenidos en tratados de libre comercio y los capítulos de protección desarrollados en acuerdos regionales de integración económica.

\footnotetext{
* Abogado. Doctor en Derecho de la Universidad de Buenos Aires, Magister en Derecho de la Universidad Nacional de Colombia y Especialista en Derecho Constitucional de la Universidad Externado de Colombia. Docente investigador de la Facultad de Derecho de la Universidad Libre de Colombia. Dirección postal: Calle 8 \#5-80. Universidad Libre, Centro de Investigaciones. Bogotá- Colombia. Dirección electrónica: jenner.tobar@unilibre.edu.co. Este trabajo es un producto de nuevo conocimiento articulado al Grupo de investigación de Derecho Privado y del proceso "Gustavo Vanegas Torres” de la Universidad Libre de Colombia.
} 
De estos, los tratados bilaterales de inversión se han convertido en el principal vehículo para la promoción y protección de la inversión extranjera, siendo la mayor parte de ellos, suscritos por países en desarrollo ${ }^{1}$.

Se estima que actualmente existen más 3000 tratados de protección a la inversión suscritos. Al menos 170 Estados han celebrados este tipo de tratados con cláusulas de arbitraje inversor-Estado o tratados de libre comercio con capítulos relativos a la protección de inversión, incluyendo Estados de altos, medios y bajos ingresos ${ }^{2}$.

Ahora bien, el gran éxito que en términos cuantitativos ha tenido estos instrumentos ha colocado en evidencia una serie de tensiones y problemáticas en todas las etapas de desarrollo de estos tratados, comenzando desde la negociación, la ejecución de las obligaciones allí contenidas y el desarrollo de los procedimientos de solución de controversias que incluyen estos tratados.

Por ejemplo, en relación con la negociación y suscripción de estos instrumentos, se ha observado que en muchas ocasiones existe una marcada asimetría de poder y experiencia entre los Estados desarrollados y los Estados en vía de desarrollo, que lleva a que los segundos acepten estos tratados sin apreciar totalmente los riesgos adquiridos y acogiendo disposiciones altamente favorables al inversor extranjero ${ }^{3}$.

Esto se debe en gran medida a que, en tiempos de liberalización económica, se ha pregonado que el libre comercio y la inversión extranjera son vehículos indispensables para alcanzar el desarrollo de las naciones, en tanto permiten al país receptor de la inversión canalizar estos recursos en beneficio de sus ciudadanos. Bajo esta lógica, los países en desarrollo entran en una competencia para ofrecer mayores ventajas competitivas a la inversión extranjera y en tal competencia, acceden a realizar amplias concesiones en asuntos como acuerdos de estabilización jurídica, beneficios tributarios y flexibilizaciones en la protección y promoción de los Derechos Humanos y las regulaciones medioambientales ${ }^{4}$.

En este contexto, en la actualidad es evidente que cuando un instrumento como un tratado de libre comercio es negociado, un capítulo de protección a la inversión y su correspondiente mecanismo de adjudicación arbitral es una condición sine qua non para que el tratado sea tomado en serio ${ }^{5}$.

Sin embargo, está lógica de expansión económica ha sido cuestionada desde al menos dos frentes. En primer lugar, la noción de desarrollo fundada exclusivamente en el ingreso ha sido ampliamente criticada en los últimos años, y en tal sentido, se ha cuestionado seriamente la capacidad de los instrumentos de protección a la inversión para generar un adecuado desarrollo, entendiendo este concepto en un sentido bastante más amplio que el simple ingreso per cápita: "Therefore, 'development must (...) be more than just the expansion of income and wealth. Its focus must be people. The concept of human development refers not only to the process of widening people's choices, but also to the level of well-being already achieved. It distinguishes between two sides of development: 'One is the formation of human capabilities, such as improved health or knowledge. The other is the use that people make of their acquired capabilities, for work or leisure"6.

Además, se ha cuestionado el efectivo potencial de los tratados de protección a la inversión para subsanar las desigualdades y propiciar el desarrollo de los estados receptores. Así, se indicado que la expansión de la economía mundial no ha tenido un impacto positivo para superar las profundas desigualdades sociales de los Estados receptores de inversión y tampoco se ha demostrado que los tratados de protección a la inversión tengan un impacto directo en la llegada de inversión extranjera, pues la existencia de un tratado de inversión es apenas uno

\footnotetext{
${ }^{1}$ AL FARUQUE (2010), p. 540.

2 BALCERZAK (2017), p. 2; SIMMA (2011), p. 574.

${ }^{3}$ SHEFFER (2011), p. 492.

${ }^{4}$ DE SCHUTTER et al (2009), p. 165.

${ }^{5}$ SWEETLAND (2016), pp. 20-21.

${ }^{6}$ DE SCHUTTER et al (2009), p. 143.
} 
entre varios factores analizados por los inversionistas al momento de decidir invertir en un Estado 7 .

Adicionalmente, algunos autores han colocado su atención en el déficit de deliberación democrática que se presenta al interior de los Estados al negociar los contenidos de estos tratados. Si bien es cierto, en las democracias representativas la negociación y suscripción de estos tratados corresponde a la autoridad gubernamental asignada para el efecto, quien en la mayoría de casos actúa procedimentalmente dentro del marco de sus competencias, en ocasiones estos procesos se adelantan de espaldas a la opinión pública y, en especial, a grupos de intereses que potencialmente se pueden ver afectados por los contenidos del tratado de inversión, así como frente a entidades gubernamentales con competencia en áreas que pueden ser eventualmente impactadas, como, por ejemplo, aquellas dependencias que regulan las políticas ambientales o laborales ${ }^{8}$.

Por otro lado, en lo que respecta al desarrollo y ejercicio de la inversión extranjera al amparo de los tratados de inversión, se han evidenciado que este ejercicio ha generado un conjunto de dificultades para el amparo efectivo de los Derechos Humanos en diferentes niveles. Así lo hizo notar desde el año 2003 el Alto Comisionado para los Derechos Humanos de Naciones Unidas, quien en el informe E/CN.4/Sub.2/2003/9 evidenció las crecientes tensiones entre ambos regímenes, así como la necesidad de introducir diferentes remedios para armonizar el desarrollo de políticas de inversión extranjera con las obligaciones de los Estados de promover y proteger los Derechos Humanos.

A continuación, se efectuará una breve contextualización en la que estas tensiones se desenvuelven el escenario internacional, a partir de lo que se conoce como fragmentación del derecho internacional. Posteriormente se efectuarán algunas consideraciones respecto a la forma como los Derechos Humanos se relacionan con los derechos usualmente reconocidos a los inversionistas extranjeros, para luego establecer los mecanismos procedimentales de integración de los Derechos Humanos dentro de los arbitrajes de inversión. Finalmente se desarrollarán algunas reflexiones de cierre con relación a los desafíos y signos de transformación que se observan en el derecho internacional de inversiones en su integración con normas de Derechos Humanos.

\section{Derechos Humanos y derecho internacional de las inversiones en contexto: la fragmentación del derecho internacional}

Desde hace varias décadas se han venido deconstruyendo los tradicionales derroteros teóricos bajos los cuales se justifica y legitima la producción normativa a nivel nacional e internacional. Bajo el análisis de los fenómenos globalizadores, se ha identificado como características definitorias de las sociedades contemporáneas la continua y creciente diferenciación funcional bajo múltiples subsistemas sociales con su propia racionalidad y autonomía.

Así, la tradicional diferenciación jerárquica del derecho en cuanto a legislación y adjudicación se ha repensado bajo heterárquicas multitudes de órdenes legales conectados estructuralmente ${ }^{9}$. En este contexto se renuncia a la premisa que el derecho adquiere exclusivamente su validez a través de los procesos de legislación y sanción estatal, y en su lugar,

\footnotetext{
${ }^{7}$ AL FARUQUE (2010), p. 551.

8 En estos escenarios resulta particularmente importante establecer los remedios preventivos que la arquitectura constitucional de cada Estado ofrece a partir del control reciproco de los poderes públicos. Un reciente ejemplo de esta situación es la que se presentó recientemente en el Estado Colombiano, donde la Corte Constitucional, en el marco de un control previo de constitucionalidad sobre el Acuerdo entre el Gobierno de la República de Colombia y el Gobierno de la República Francesa sobre el Fomento y Protección Recíprocos de Inversiones, estableció la existencia de varios reparos de constitucionalidad sobre el Acuerdo, por lo cual instó al gobierno colombiano a negociar con su contraparte francesa, la adopción de una declaración interpretativa conjunta que subsane los deficits adevertidos por la Corte (Corte Constitucional de Colombia, Sala plena, Sentencia C-252, 2019). Confróntese: CHOUDHURY (2009), pp. 1000-1002.
}

9 TEUBNER (1997), p. 777. 
se ha establecido una fragmentación de la sociedad mundial, la cual propicia la aparición de estas nuevas formas heterárquicas de derecho en varios sectores de la sociedad ${ }^{10}$.

En el área del derecho internacional, estas dinámicas de fragmentación han operado en diversos ámbitos. En primer lugar, resulta evidente que, bajo procesos autorregulatorios, los particulares desarrollan continuas dinámicas de producción normativa en los diversos subsectores, compartiendo así el proceso de racionalización legal junto con los Estados ${ }^{11}$. En segundo lugar, como resultado de la especialización funcional, se han creado regímenes especiales en áreas de experticia como derecho mercantil internacional, derecho internacional ambiental, derecho penal internacional, derecho internacional de los Derechos Humanos, derecho internacional de las inversiones, entre otros ${ }^{12}$.

Si bien el desarrollo de estos escenarios de especialización surge como consecuencia lógica de la complejidad propia de las sociedades modernas, resulta problemático cuando no se desarrollan también mecanismos de irritación e interpenetración entre los diversos subsistemas especializados, o cuando los mecanismos existentes resultan deficientes.

Históricamente el derecho corporativo y el Derecho Internacional de los DD.HH se han desarrollado de forma separada uno de otro ${ }^{13}$. Así, durante décadas el derecho internacional de inversiones permaneció ajeno a la influencia del derecho internacional de los Derechos Humanos en tanto los tratados de inversión no suelen desarrollar provisiones destinadas a la protección de los Derechos Humanos ${ }^{14}$.

Empero, la deficiencia de responsabilidades de los empresarios ha generado que se abra todo un dialogo entre el sector empresarial y los demás sectores de la sociedad ${ }^{15}$ en el que se han generado diversas dinámicas de observancia voluntaria de estándares e incluso la implementación de contenidos de DD.HH en algunos tratados de inversión. En este sentido, en el conocido informe Ruggie sobre Derechos Humanos y Empresas Transnacionales (2008) se señaló que:

"Para atraer inversiones extranjeras, los Estados de acogida ofrecen protección por medio de
tratados bilaterales de inversión y acuerdos de gobiernos receptores. Estos prometen tratar a los
inversores de manera justa, equitativa y sin discriminación, y se comprometen a no hacer cambios
unilaterales en las condiciones de inversión. Ahora bien, las protecciones al inversor han aumentado
sin tener mucho en cuenta los deberes estatales de proteger los derechos humanos, sesgando el
equilibrio entre ambos aspectos. Por consiguiente, a los Estados de acogida les puede resultar difíil
fortalecer las normas nacionales en el plano social y ambiental, en particular las relacionadas con
los derechos humanos, sin temor de las impugnaciones de inversores extranjeros que pueden
plantearse en virtud de arbitrajes internacionales vinculantes" ${ }^{16}$.

No obstante, se ha establecido que los tribunales de inversión suelen ser normalmente reacios a admitir argumentos de Derechos Humanos dentro de sus procedimientos, ya sean presentados directamente por las partes o por interesados a través de amicus curiae, pero así mismo, dentro del sistema internacional de Derechos Humanos ha existido reticencia a reconocer la influencia que reclamos paralelos bajo el mismo conjunto de hechos podría tener o no en sus decisiones ${ }^{17}$. En cada caso, el fallador termina resolviendo el conflicto sobre la base exclusiva de un instrumento internacional específico del cual deriva su jurisdicción ${ }^{18}$.

${ }^{10}$ SALDIVIA (2010), p. 39; TOBAR (2017), p. 81.

11 TOBAR (2017), p. 104.

12 KOSKENNIEMI (2011), p. 65.

13 STEINHARDT (2005), p. 177.

${ }^{14}$ BALCERZAK (2017), p. 3.

${ }^{15}$ CAFAGGI (2015), p. 924.

${ }^{16}$ CONSEJO DE DD.HH (2008), p. 12.

17 POLANCO (S.F), p. 21; HIRSCH (2009), p. 107.

18 Un ejemplo ilustrativo de ello es el caso Bernhard von Pezold y otros vs Republica de Zimbabwe. En este caso se resolvió de forma previa una solicitud de intervención amicus curiae del Centro Europeo de Derechos Constitucionales y Humanos y 4 comunidades indígenas de Zimbawe donde se solicitaba, entre otros, que el tribunal tomara en consideración los derechos internacionales reconocidos a los pueblos indígenas sobre sus territorios y el impacto que la decisión podría tener sobre ellos. Al resolver la solicitud, el tribunal arbitral consideró que no estaba demostrado que el derecho internacional de inversiones y el derecho internacional de los 
Estos escenarios de fragmentación solamente podrán ser superados si se renuncia a una lectura de la situación en clave de jerarquización, y en su lugar abordar el problema bajo lógicas de integración funcional. En este sentido Teubner propone dar un giro en la forma como se abordan los conflictos jurídicos internacionales, para pasar de estos a conflictos entre regímenes ${ }^{19}$. Toda vez que es común que los conflictos transnacionales no puedan atribuirse a un único derecho nacional, es necesaria la creación de nuevas normas de conflicto que no elijan el derecho aplicable entre derechos nacionales sino entre regímenes funcionales y desarrollar normas sustantivas que respondan a esta lógica.

Bajo esta misma perspectiva, Feria- Tinta considera que existen 4 elementos clave para desarrollar lo que denomina "el principio de integración del derecho internacional". Señala esta autora: "These are: (1) conflict resolution and interpretation cannot be distinguished from each other; (2) international law is understood as a system; (3) all international law exists in a systemic relationship with other law (therefore in interpreting a rule one ought to look into the normative environment of a treaty); and (4) a limited jurisdiction does not imply a limitation of the scope of the law applicable in the interpretation and application of the treaty"20.

De este modo, bajo una comprensión en clave sistémica del derecho internacional, no se asume la existencia de diversos regímenes, sino que los conflictos se abordan y resuelven bajo el derecho internacional como un único sistema. Esta idea ha sido presentada y defendida en el Reporte del grupo de estudio de la Comisión de Derecho Internacional de Naciones Unidas (2006), donde se indicó que si bien es cierto un tribunal deriva su competencia de un instrumento en particular, tiene el deber de interpretar y aplicar los contenidos sustantivos de dicho instrumento en relación con su entorno normativo, es decir, con los demás instrumentos de derecho internacional relevantes, deber que se desprende del artículo 31 de la Convención de Viena sobre el Derecho de los Tratados ${ }^{21}$.

Sin embargo, la práctica arbitral ha mostrado que esta compresión en clave sistémica del derecho internacional de las inversiones y de los DD.HH ha encontrado varias dificultades y limitaciones en diversas cuestiones. A continuación, se desarrollarán con cierto detalle algunos de los aspectos más discutidos en torno a la receptividad y resistencias del discurso de los Derechos Humanos en los arbitrajes de inversión.

\section{El inversionista extranjero y su relación con los derechos humanos. Una relación de ida y vuelta}

\subsection{Los derechos de los inversionistas como Derechos Humanos}

Históricamente los Derechos Humanos se han comprendido como garantías con diverso contenido, todas dirigidas a la protección de los individuos en nuestra naturaleza de seres humanos, no extensible a priori a las personas jurídicas. Sin embargo, en diversas jurisdicciones nacionales e internacionales, las corporaciones han sido sujetos de reconocimiento paulatino de

Derechos Humanos fueran interdependientes y que estuviere obligado a tener en consideración los segundos para una decisión, por lo cual el tribunal deliberadamente omitió tomar en consideración instrumentos internacionales de Derechos Humanos respecto a pueblos indígenas, al considerar que su mandato estaba expresamente circunscrito al tratado bilateral de inversión aplicable al caso concreto. En esta misma línea se han proferido otras decisiones donde el tribunal arbitral ha apuntado hacia la falta de competencia para atender reclamos o asuntos relacionados con los Derechos Humanos, o para utilizar los instrumentos que consagran tales obligaciones, entre ellas encontramos los casos Biloune v Ghana, Channel Tunnel Group vs Reino Unido y Francia, Rompetrol Group vs Rumania, Quasar de Valores SICAV S.A. y otros vs Federación de Rusia. En otras decisiones el tribunal arbitral ha optado por ignorar o evadir los argumentos de Derechos Humanos planteados por el Estado o terceros, ejemplos de ellos son los casos United Parcel Services of America Inc. vs Canadá, Glamis Gold, Ltd vs Estados Unidos.

19 TEUBNER (2005), p. 123.

${ }^{20}$ FERIA- TINTA (2017), p. 608.

${ }^{21}$ En el procedimiento de anulación del caso Tulip Real Estate And Development Netherlands B.V vs Turquia el Tribunal de anulación respaldó expresamente el principio de integración sistémica bajo el desrarollo del artículo 31.3.c de la Convención de Viena, así: “The ILC has discussed Article 31(3)(c) of the VCLT extensively in its Fragmentation Report. In doing so, its Study Group has referred to that provision as a "master key" to the house of international law. (...) Provisions in human rights instruments dealing with the right to a fair trial and any judicial practice thereto are relevant to the interpretation of the concept of a fundamental rule of procedure as used in Article 52(1)(d) of the ICSID Convention. This is not to add obligations extraneous to the ICSID Convention. Rather, resort to authorities stemming from the field of human rights for this purpose is a legitimate method of treaty interpretation". 
ciertos derechos civiles como el debido proceso, igualdad, libertad de conciencia, libertad de expresión, entre otros ${ }^{22}$.

Como una respuesta para superar los escenarios de fragmentación en el derecho internacional, específicamente entre el Derecho internacional de los Derechos Humanos y el derecho internacional de inversiones, se ha propuesto una relectura convergente de ambos escenarios para proponer que la protección de la inversión extranjera puede ser entendida como parte del Derecho internacional de los Derechos Humanos, conclusión a que se ha llegado al analizar que ambos derechos tienen más semejanzas sustantivas que diferencias ${ }^{23}$.

Si bien es evidente que existen diferencias entre ambos catálogos de derechos (por ejemplo, el criterio de extranjería es elemental en el derecho internacional de inversiones, mientras que en el campo de los DD.HH su protección se irradia a nacionales y foráneos), también se pueden identificar varias semejanzas, como, por ejemplo, que ambos catálogos de derechos surgen como mecanismos de protección de las personas -naturales o jurídicas- ante las violaciones $o$ arbitrariedades que pudieren cometer las autoridades públicas ${ }^{24}$.

Mas relevante aun son las semejanzas entre los contenidos sustantivos de ambos regímenes. La protección contra la expropiación, figura propia del derecho internacional de inversiones, encuentra un soporte evidente en el respeto a la propiedad privada, este último reconocido por varios instrumentos regionales de protección a los Derechos Humanos como el primer protocolo de la Convención Europea sobre Derechos Humanos (articulo 1), la carta de Banjul (artículo 14) o la Convención Americana de Derechos Humanos (artículo 21), entre varios otros instrumentos semejantes ${ }^{25}$. Si bien el alcance e interpretación respecto a la protección de la propiedad como un derecho humano no ha sido uniforme, se puede anotar, como lo hace Klein (2012), que al menos un mínimo estándar de protección a la propiedad puede ser entendido como un derecho humano universal.

Por su parte, el estándar de trato justo y equitativo del derecho internacional de inversiones, encuentra respaldo en los derechos universales a la igualdad, prohibición de discriminación y debido proceso, cuyos contenidos pueden ayudar a interpretar el estándar de trato justo y equitativo ${ }^{26}$.

El estándar de total protección y seguridad puede ser asociado a los contenidos que se han desarrollado alrededor del derecho humano de libertad personal y seguridad, consignado en el Pacto Internacional de Derechos Civiles y Políticos. De considerarse garantías análogas, bien podría pensarse que una violación al deber del Estado de proveer seguridad al inversionista podría configurar una transgresión a ese instrumento de Derechos Humanos ${ }^{27}$. De hecho, este enfoque fue adoptado por el tribunal arbitral en el caso Hesham T. M. Al Warraq vs Indonesia, donde el Tribunal acudió a los derechos reconocidos por el Pacto, para reforzar el Demandante no recibió un trato justo y equitativo.

A pesar de estas semejanzas, la práctica ha mostrado que pocas veces los inversionistas efectúan sus reclamos solicitando la protección de los Derechos Humanos, sino que acuden a los estándares de protección del derecho internacional de inversiones. Esto se puede explicar por varios factores: primero son pocos instrumentos de Derechos Humanos los que reconocen protección expresa a las personas jurídicas, además existe la idea de que los estándares de protección que brindan los instrumentos de Derechos Humanos, son más restringidos que los estándares de los instrumentos de protección a la inversión y a todo ello se suma la reticencia de los tribunales de inversión para aplicar instrumentos diferentes al tratado del cual derivan su competencia ${ }^{28}$.

\footnotetext{
${ }^{22}$ Sobre este tema se pueden consultar las obras de GREENFIELD (2018) y WINKLER (2018), quienes efectúan un detallado análisis del reconocimiento de derechos civiles a las corporaciones bajo el derecho estadounidense.

${ }^{23}$ KLEIN (2012), p. 181

${ }^{24}$ KLEIN (2012), pp. 187-188.

${ }^{25}$ KLEIN (2012), pp. 189-190.

${ }^{26}$ LO (2015), p. 9.

${ }^{27}$ LO (2015), pp. 8.

${ }^{28}$ REINER Y SCHREUER (2009), p. 88.
} 
Ejemplo de esto se puede encontrar en el caso Houben vs Burundi, donde se discutió un caso relativo a la protección a la propiedad del inversor (Sr. Houben) sobre un terreno de 14 hectáreas, en las cuales se presentaron ventas y ocupaciones ilegales, aparentemente toleradas por el Estado de Burundi. Si bien, en un caso como estos hubiese sido de suma pertinencia que el tribunal se pronunciara respecto a la procedencia de la protección garantizada por los Instrumentos de Derechos Humanos aplicables, el Tribunal se declaró incompetente para ello y centró su análisis estableciendo la violación o no del tratado bilateral de inversión aplicable al caso. En otros casos como Bozbey vs Turkmenistán o Mykhailenko vs Bielorrusia, si bien los hechos que dieron lugar a la disputa tenia clara relación con graves violaciones a los Derechos Humanos del inversor, los tribunales arbitrales no se pronunciaron al respecto pues la controversia fue dirimida por acuerdo anticipado entre las partes ${ }^{29}$.

De este modo, la aproximación conceptual que se busca realizar entre Derechos Humanos y derechos del inversionista está dirigida a eliminar estas brechas que la práctica del derecho internacional ha generado entre ambos regímenes.

\begin{abstract}
"Understanding investment protection as one right among others that first needs to be understood as minimum standard and second, needs to be balanced with other human rights may serve investment tribunals in the future as conceptual tool to effectively balance investment protection with other human rights. If this understanding was taken seriously, the system of IIL could hardly be viewed as illegitimate and IIL would not represent a threat to international human rights protection but rather a useful supplement to IHRL, designed to promote economic growth, the protection of property and related economic rights. While it cannot be said that existing IIL standards will be transferred into future human rights standards one-toone, one should be mindful that as a tendency under international law rights of foreigners, as long as they constitute minimum standards, may eventually become accepted as rights for all. In any event, there exists considerable potential for cross-fertilization between the two fields of law" ${ }^{\prime 30}$.
\end{abstract}

\title{
2.2. La obligación de los inversionistas de respetar los Derechos Humanos
}

Desde el S. XVIII los DD.HH han sido un concepto en continua evolución. Originalmente se plantearon como garantías de corte individual cuyo fin central era proteger al individuo de la intromisión del Estado, posteriormente se reconoció que para la efectividad real de muchos de estos derechos era necesaria una posición activa del Estado e incluso se ha establecido la existencia de ciertos DD.HH que tienen impacto directo en el desarrollo de las sociedades consideradas en su conjunto ${ }^{31}$.

Durante décadas se ha considerado que por su propia naturaleza y génesis los DD.HH son garantías que se pueden exigir respecto al Estado pero no frente a los particulares. No obstante, en atención al gran poder que los grupos privados ostentan en varios sectores de la economía, han surgido voces que reclaman la eficacia horizontal de los DD. HH y su consecuente exigibilidad entre particulares, siendo pioneros en este tema la doctrina alemana a través de la denominada "Drittwirkung". A grandes rasgos, esta teoría defiende la aplicación de los DD.HH entre particulares reconociendo la asimetría fáctica entre los mismos y el gran poder de hecho que varios particulares ejercen en las relaciones modernas.

Bajo este enfoque son numerosas las voces que han reclamado la creación y desarrollo de obligaciones de los particulares respecto a su observancia de los Derechos Humanos, aspecto especialmente relevante en la inversión extranjera, cuyas actividades económicas muchas veces tienen un impacto significativo en la población local. De esta forma, se pueden identificar por lo

\footnotetext{
${ }^{29}$ BALCERZAK (2017), p. 73.

${ }^{30}$ KLEIN (2012), p. 195.

${ }^{31}$ Múltiple bibliografia puede ser referenciada respecto a los DD.HH y las denominadas "generaciones de derechos". Por cuestiones de espacio, vale la pena apenas referenciar la sentencia de la Corte Constitucional de la República de Colombia (1 de abril de 2014) Sentencia T-197 de 2014. Allí esta corporación plantea reglas jurisiprudenciales sobre la exigibilidad de cada una de estas tipologias de DD.HH y su relación con la Constitución Política.
} 
menos dos vías a través de las cuales se ha exigido el cumplimiento de estos estándares a los inversores.

En primer lugar, el mecanismo más acogido por los actores privados del comercio internacional para comprometerse con el respeto de los Derechos Humanos es aceptar mecanismos legales no vinculantes y de cumplimiento voluntario, o en otras palabras, a través de instrumentos de soft law.

En tal sentido, varios tratados bilaterales de inversión han incluido estándares de cumplimiento voluntario a cargo del inversionista sobre la protección de Derechos Humanos, laborales, medioambientales, sociales o culturales, por lo cual los deberes del inversionista quedan circunscritos a cláusulas de responsabilidad social corporativa, como es el caso de tratados bilaterales de inversión como los celebrados entre Canadá-Benín (artículo 16), BrasilMozambique (artículo 10), tratados de libre comercio con capítulos de inversión como el suscrito entre Colombia- Canadá (artículo 816), o instrumentos regionales como el Protocolo de Cooperación y Facilitación de Inversiones Intra-Mercosur (artículo 14), entre otros.

En este contexto, los Códigos de conducta empresarial, como manifestación autorregulatoria, han experimentado una creciente relevancia en virtud de lo que se ha conocido como ética de los negocios o ética empresarial en donde la responsabilidad social empresaria ha ocupado un papel esencial en el desarrollo productivo empresarial y su supervivencia en el mercado. Sin embargo, a pesar del auge en su implementación por las empresas y asociaciones empresariales, estos instrumentos autorregulatorios han sido cuestionados por sus limitaciones, entre las que se destaca la ausencia de un monitoreo independiente que garantice que no son simples declaraciones de principios empresariales, la poca participación en su elaboración de los interesados directos -trabajadores, asociaciones ambientales, etc.-, entre otros.

Específicamente en el escenario internacional de inversiones, la ausencia de disposiciones sustantivas respecto a obligaciones de Derechos Humanos dentro de los tratados de inversión, así como la inexistencia de un tratado vinculante sobre Derechos Humanos y Empresas transnacionales ${ }^{32}$, sumado al carácter voluntario de los estándares autorregulatorios adoptados, han llevado a que en varias ocasiones los tribunales arbitrales resuelvan las disputas de inversiones exclusivamente con base en las interpretaciones que se realizan de los estándares de protección de inversión contenidos en el respectivo tratado, y a partir de interpretaciones flexibles frente a la existencia de obligaciones reales a cargo del inversor.

En este sentido, autores como De Brabandere o Dumberry \& Dumas-Aubin ${ }^{33}$ resaltan la ausencia de obligaciones directas y estrictamente vinculantes a cargo de los particulares frente al respeto y observancia de los Derechos Humanos en el marco de controversias de derecho internacional de las inversiones, ausencia derivada de la inexistencia de un instrumento internacional vinculante en tal sentido. Por tal motivo se ha indicado que suele existir una marcada asimetría entre la protección del inversor y la del Estado, en tanto usualmente los derechos del primero están cuidadosamente detallados, se omiten obligaciones derivadas del sistema internacional de Derechos Humanos a cargo del segundo ${ }^{34}$.

\footnotetext{
${ }^{32}$ A finales de junio de 2014, durante la 26 sesión del Consejo de Derechos Humanos de la ONU en Ginebra, se adoptó una importante decisión: "establecer un grupo de trabajo intergubernamental de composición abierta sobre las empresas transnacionales y otras empresas con respecto a los Derechos Humanos, cuyo mandato es elaborar un instrumento jurídicamente vinculante para regular las actividades de las empresas transnacionales y otras empresas en el DIH" CONSEJO DE DERECHOS HUMANOS DE LAS NACIONES UNIDAS (2014), p. 2. Desde entonces se realizan reuniones de trabajo anuales, la cuarta sesión de discusiones se realizó en octubre de 2018, donde se comenzó el examen de la propuesta de articulado del instrumento vinculante. La sexta sesión se desarrolló en octubre de 2020.

${ }^{33}$ DUMBERRY Y DUMAS-AUBIN (2012), p. 350; DE BRABANDERE (2018), p. 619.

${ }^{34}$ HERNANDEZ Y RAMIRO (2015), p. 15.
} 


\section{3. ¿Cómo pueden utilizarse los DD.HH en las controversias de inversión? Vías procedimentales de acceso}

Los debates alrededor de la posibilidad de tomar en cuenta reglas provenientes del derecho internacional de los Derechos Humanos al interior de los arbitrajes de inversión, se han desarrollado a partir de controversias procedimentales donde se han discutido alrededor de las normas que permiten a los árbitros aplicar reglas de Derechos Humanos en las controversias de inversiones.

Respecto a la discusión procedimental, en la doctrina y en los casos de arbitraje se han identificado al menos 4 vías a través de las cuales las reglas de Derechos Humanos pueden ser incorporadas como derecho de fondo en los procedimientos arbitrales de inversión, a saber, i) bajo la noción de reglas de derecho internacional, ii) a través del principio de integración sistémica del derecho de los tratados, iii) como contenido del derecho local del Estado receptor de la inversión y iv) mediante el desarrollo de la noción de orden público internacional.

En primer lugar, se debe señalar que Convenio CIADI no contiene reglas que determinen estrictamente el derecho aplicable a las disputas de inversión. El artículo 42.1 del Convenio establece que "El Tribunal decidirá la diferencia de acuerdo con las normas de derecho acordadas por las partes. A falta de acuerdo, el Tribunal aplicará la legislación del Estado que sea parte en la diferencia, incluyendo sus normas de derecho internacional privado, y aquellas normas de derecho internacional que pudieren ser aplicables". Con alguna semejanza, el Tratado de Libre Comercio de América del Norte (TLCAN) señala en su artículo 1131.1 que "un tribunal establecido conforme a esta sección decidirá las controversias que se sometan a su consideración de conformidad con este Tratado y con las reglas aplicables del derecho internacional". Por su parte, el Tratado de la Carta de Energía indica en su artículo 26.6 que "se creará un tribunal que decidirá las cuestiones en litigio con arreglo al presente Tratado y a las normas del Derecho Internacional aplicables" (subrayados fuera del texto original).

Estas normas han sido interpretadas como una primera vía de acceso procedimental para que los tribunales arbitrales tomen en cuenta las reglas del derecho internacional de Derechos Humanos, al momento de resolver la controversia. Por supuesto, se evidencia en los tres casos mencionados que al Tribunal arbitral se le concede un amplio margen de apreciación para establecer si determinada regla del derecho internacional tiene o no relevancia en la disputa, bajo criterios que el mismo tribunal determine. Así mismo, corresponde al tribunal establecer la relación de complementariedad o subsidiariedad entre el derecho internacional y el derecho interno y la forma como resuelve los posibles conflictos entre uno y otro, evidenciándose que en esta hipótesis suele darse prevalencia al derecho internacional sobre el derecho interno ${ }^{35}$.

Por tanto, bajo esta vía, las reglas de Derechos Humanos solo serán tenidas en cuenta en las controversias de inversión en la medida que el tribunal respectivo las considere pertinentes para el caso en cuestión, agregando, además, que en el caso del Convenio CIADI, la redacción de la norma permite interpretar que esta facultad del Tribunal es supletiva, es decir que operará sólo en caso que las partes no alcancen un acuerdo sobre el derecho aplicable.

Una segunda vía procedimental para la aplicación de los Derechos Humanos en arbitrajes de inversión se ha desarrollado a partir del denominado principio interpretativo de integración sistémica contenido en el artículo 31.3.c de la Convención de Viena sobre el derecho de los tratados que señala que "Un tratado deberá interpretarse de buena fe conforme al sentido corriente que haya de atribuirse a los términos del tratado en el contexto de estos y teniendo en cuenta su objeto y fin. (...) 3. Juntamente con el contexto, habrá de tenerse en cuenta: (...) c) toda forma pertinente de derecho internacional aplicable en las relaciones entre las partes".

Si bien esta norma recoge un criterio a tomar en cuenta al momento de resolver controversias que involucren el desarrollo de tratados internacionales -como es el caso de las controversias de inversiones-, los alcances de su aplicación rebosan el escenario meramente

\footnotetext{
${ }^{35}$ LINARES (2019), p. 429.
} 
interpretativo y se torna en un auténtico criterio que indica al fallador el contexto normativo concreto en el cual el tratado respectivo debe ser aplicado en concordancia con el principio de buena $\mathrm{fe}^{36}$.

De este modo, al utilizar esta general de derecho internacional público, los tribunales deberían establecer si las normas de Derechos Humanos resultan pertinentes para la controversia en cuestión y determinar si dicha regla resulta aplicable en las relaciones entre las partes. En términos generales se puede afirmar que normas internacionales en materias claves en el desarrollo de inversiones como temas medioambientales, laborales, de seguridad social y salud son pertinentes en la resolución de disputas de inversiones y deben ser utilizadas como herramientas de interpretación e integración ${ }^{37}$.

No obstante, se ha cuestionado si en estos contextos se puede afirmar que una norma de Derechos Humanos es estrictamente aplicable a los particulares que conforman una de las partes en las controversias de inversión ${ }^{38}$. Bajo una lectura restringida y fragmentada, las obligaciones de Derechos Humanos están a cargo exclusivamente de los Estados, y en tal sentido este argumento podría llevar a considerar que normas de Derechos Humanos no cabrían bajo los presupuestos de aplicación señalados en 31.3.c de la Convención de Viena, al no ser aplicables directamente a particulares.

Sin embargo, una lectura detenida de la norma en cuestión muestra que para aplicar normas de derechos humanos como "forma pertinente de derecho internacional" se requiere que estas puedan ser aplicadas en "las relaciones" entre las partes, lo que se puede sostener, por lo menos, en aquellos casos en los cuales el Estado inversor ha adquirido obligaciones de Derechos Humanos con anterioridad a la suscripción del tratado bilateral de inversión, pues en tales casos el inversor conoce de antemano que tales normas conforman el ambiente regulatorio que le afecta. Así mismo, pude defenderse por esta vía, la aplicación de normas de Derechos Humanos que sean consideradas como normas de ius cogens, en tanto la operatividad de estas disposiciones irradia la actividad de los Estados y los particulares en todo contexto ${ }^{39}$.

Una tercera vía procedimental a través de la cual normas de Derechos Humanos pueden ser incorporadas a los procedimientos arbitrales de inversión es mediante la aplicación del derecho local del Estado parte en la controversia. En la mayoría de los casos, el derecho interno de los Estados receptores de inversión establece una relación directa entre sus normas constitucionales y ciertas normas de derechos humanos provenientes de tratados ratificados por el respectivo Estado.

Si bien es cierto, los mecanismos a través de los cuales se incorporan las normas internacionales de Derechos Humanos al ordenamiento interno pueden variar de un Estado a otro, el ambiente regulatorio del Estado receptor es un elemento que el potencial inversor deberá tener en cuenta al momento de llevar su inversor a dicho Estado, especialmente respecto a requerimientos constitucionales o interpretaciones judiciales vigentes de normas internas sobre Derechos Humanos, que potencialmente puedan afectar el desarrollo de la inversión. Si este marco regulatorio es conocido de antemano por el inversor, tal situación influirá necesariamente en sus "legítimas expectativas" y como desarrollo del principio de buena fe

\footnotetext{
${ }^{36}$ DE BRABANDERE (2018), p. 638; FERIA (2017), p. 620.

37 LINARES (2019), p. 522.

38 SIMMA (2011), p. 585.

${ }^{39}$ Este enfoque fue respaldado en el caso Urbaser vs Argentina, en el cual el tribunal, además de aludir a la pertinencia de la normas de derecho internacional a partir del artículo 41.2 del Convenio CIADI, y del concepto de normas de ius cogens, indicó expresamente que el tratado de inversión "debe interpretarse a la luz de las normas establecidas en la Convención de Viena sobre el Derecho de los Tratados del 23 de mayo de 1969 y que el Artículo 31, inciso 3(c) de ese Tratado indica que debe tenerse en cuenta "toda forma pertinente de derecho internacional aplicable en las relaciones entre las partes". EI TBI no puede interpretarse y aplicarse de manera aislada. El Tribunal debe tener claramente presente el objetivo especial del TBI como un Tratado que promueve las inversiones extranjeras, pero no puede hacerlo sin tener en cuenta las normas relevantes de derecho internacional. EI TBI debe interpretarse en armonía con otras normas de derecho internacional de las que forma parte, incluidas aquellas relativas a los derechos humanos". Una aproximación similar se dio dentro del el procedimiento de anulación del caso Tulip Real Estate And Development Netherlands B.V vs Turquia, atrás citado.
} 
debería estar compelido a respetar las normas de derecho interno ${ }^{40}$, sin que ello signifique que el Estado pueda oponer sus normas de derecho interno para desconocer las obligaciones internacionales que adquiera con ocasión de los tratados internacionales de inversión que celebre $^{41}$.

Finalmente, como cuarta formula de acceso de los Derechos Humanos al interior de los arbitrajes de inversión se ha planteado el desarrollo en estos escenarios del concepto de "orden público transnacional", noción más desarrollada en el arbitraje comercial internacional que en el arbitraje de inversión ${ }^{42}$.

Quienes defienden la aplicación de normas de orden público transnacional o verdaderamente internacional resaltan la importancia y necesidad de establecer un conjunto de reglas y principios que "recojan las normas de conducta, estándares éticos y valores fundamentales compartidos por la gran mayoría de sistemas legales, siendo verdaderamente internacional en tanto no está ligado o emana de un sistema legal en particular, sino que se conforma por valores fundamentales universales que deben ser aplicados de manera obligatoria para mantener mínimos de conducta en las transacciones internacionales" ${ }^{43}$. Autores como Linares encuentran en esta figura una posible "válvula de escape" que puede ser utilizadas por los Estados, cuando se requiere el reconocimiento y ejecución de una decisión extranjera, para ponderar el cumplimiento de principios generales del derecho que conforman el "orden público internacional" del Estado donde se busca la ejecución de la de la decisión arbitral ${ }^{44}$.

Sin embargo, el desarrollo práctico de este concepto encuentra varias limitaciones relacionadas principalmente con la dificultad que los árbitros enfrentan al establecer que principios pueden ser considerados como "universales" y cómo establecer el grado de aceptación que tal norma debe tener en el ámbito internacional ${ }^{45}$. En el escenario específico del arbitraje de inversiones, los árbitros podrían analizar este concepto tomando como punto de partida inicial la aceptación del principio bajo estudio en los Estados parte del tratado que les da competencia.

Como lo referencia Dupuy, dentro del arbitraje de inversiones ya ha sido desarrollada la noción de orden público transnacional ${ }^{46}$. Específicamente en el caso WORLD DUTY VS KENYA, el Tribunal arbitral estableció que "in light of domestic laws and international conventions relating to corruption, and in light of the decisions taken in the matter by courts and international tribunals, this Tribunal is convinced that bribery is contrary to the international public policy of most, if not all States, or to use another formula, to transnational public policy. Thus, claims based on contracts of corruption or on contracts obtained by corruption cannot be upheld by this Arbitral Tribunal".

\footnotetext{
${ }^{40}$ Esta posición fue adoptada por el Tribunal arbitral del caso Philip Morris Brands Sàrl, Philip Morris Products S.A.y Abal Hermanos S.A. vs Uruguay donde el tribunal estableció que el Estado uruguayo había desarrollado una regulación sobre el consumo del tabaco, acorde a sus normas de derecho interno y compromisos internacionales en la materia. Además, respecto a la facultad de regular temas de orden público, como la salud y la noción de legítimas expectativas, se señaló que "Se desprende claramente del análisis del estándar de TJE realizado por tribunales en casos de inversión, que las expectativas legítimas dependen de compromisos y declaraciones de naturaleza específica del Estado receptor, expresadas para inducir a los inversores a realizar inversiones. Las disposiciones de legislación general aplicables a una pluralidad de personas, o de categorías de personas, no crean expectativas legítimas de que no habrá cambios en la legislación. Dados los poderes regulatorios del Estado, para basarse en las expectativas legítimas, un inversor debería consultar con antelación respecto de las perspectivas de un cambio en el marco regulatorio a la luz de los cambios vigentes o razonablemente esperables de las condiciones económicas y sociales del Estado receptor".

${ }^{41}$ DUPUY (2009), pp. 58-59.

42 DUPUY (2009), p. 59.

43 TOBAR (2017), p. 370.

${ }^{44}$ LINARES (2019), pp. 557-561.

${ }^{45}$ BARRACLOUGH Y WAINCYMER (2005), p. 219.

${ }^{46}$ DUPUY (2009), p. 60.
} 


\section{Corolario. Desafíos y signos de transformación en el derecho internacional de inversiones. El derecho de los Estados a regular como ejemplo de este panorama}

Durante el desarrollo de este texto se pretendió ilustrar dos premisas: la primera es que el desarrollo actual del derecho de inversiones refleja un marcado escenario de fragmentación y déficit respecto al necesario dialogo e interacción que esta rama del derecho internacional debe tener con el Derecho Internacional de los Derechos Humanos.

La segunda premisa que se desarrolló es que esto se debe, en parte, a las limitadas vías de acceso que el derecho internacional de inversiones ofrece para la aplicación directa de los Derechos Humanos en los arbitrajes de inversión. Las interacciones hasta ahora existentes han resultado insatisfactorias en la medida que los instrumentos de protección a la inversión no suelen acoplar en forma vinculante reglas de protección a los Derechos Humanos, tendencia que se ha reflejado en la decisiones arbitrales donde suelen prevalecer los argumentos derivados de instrumentos de inversión, y solo excepcionalmente se han tomado en cuenta para el fondo de la decisión normas de Derechos Humanos, incorporadas a la controversia mediante los mecanismos interpretativos atrás expuestos.

Sin embargo, como resultado de la agenda pública en donde se ha establecido deficiencia obligacional respecto a los DD.HH en el marco del derecho internacional de inversiones, hoy los Estados son conscientes de esta situación y en tal sentido deben tomar nota para lograr que los instrumentos de inversión suscritos reflejen un mayor compromiso de los particulares en el desarrollo de sus actividades.

En tal sentido, multiplicidad de actores estatales e internacionales han resaltado la necesidad de efectuar cambios en el sistema internacional de inversiones para garantizar ciertos mínimos de protección en torno a los Derechos Humanos. Un ejemplo de esto es el desarrollo que se ha dado al problema de limitación de la capacidad regulatoria de los Estados derivada de las obligaciones adquiridas en tratados de inversión. Como se desarrollará, al respecto se han comenzado a generar lentas transformaciones en diversos niveles para superar este déficit que impacta en el gozo de los Derechos Humanos de los habitantes del Estado anfitrión.

Como se expuso anteriormente, en muchas ocasiones los tratados de Inversión contienen cláusulas y disposiciones a favor del inversor con una clara textura abierta o acudiendo a conceptos jurídicos indefinidos, de tal forma que el contenido concreto de las obligaciones que los Estados adquieren a través de los tratados de protección, ha sido establecido en varias ocasiones ex post por medio de los fallos arbitrales.

En este contexto, los Estados se enfrentan a verdaderos dilemas cuando consideran necesario intervenir mediante regulación en un escenario donde el inversor está desarrollando sus actividades, pues debe considerar detenidamente si la regulación que pretenden puede colocar en riesgo los derechos que el tratado de inversión ha otorgado al inversionista extranjero.

Este ha sido uno señalado como uno de los puntos de tensión más recurrentes entre Derechos Humanos y derecho de la inversión, en tanto, ante la posibilidad que la regulación estatal sea vista por el inversor como una interferencia indebida en el ejercicio de sus derechos, los Estados pueden optar por abstenerse de regular el ámbito respectivo, con el consecuente detrimento de los derechos humanos de los habitantes. Este fenómeno se ha conocido como "enfriamiento regulatorio" y ha sido advertido en el Informe del Representante Especial del Secretario General de la ONU sobre Derechos Humanos y empresas transnacionales, que en el año 2009 señaló:

\footnotetext{
"Recent experience suggests that some treaty guarantees and contract provisions may unduly constrain the host Government's ability to achieve its legitimate policy objectives, including its international human rights obligations. That is because under threat of binding international arbitration, a foreign investor may be able to insulate its business venture from new laws and regulations, or seek compensation from the Government for the cost of compliance".
} 
Los peligros del enfriamiento regulatorio no solamente recaen en los efectos nocivos que en la ciudadanía puede tener que un Estado se abstenga de intervenir para corregir o superar déficits de protección en DD.HH sino que puede generar que el inversionista desafíe regulaciones de Derechos Humanos nacionales o internacionales que -a su juicio- afectan sus intereses, lo que colocaría al Estado en una situación sin salida donde debe elegir si opta por dar prevalencia a sus compromisos de protección de los $\mathrm{DD} . \mathrm{HH}$, caso en el cual tendrá que involucrarse en un seguro litigio con el inversor y eventualmente asumir responsabilidad por violaciones a los tratados de inversión, u opta por dar prevalencia a estos últimos, caso en el cual sacrificará el bienestar de sus ciudadanos ${ }^{47}$.

Lo anterior es especialmente evidente en estándares de protección a la inversión como la expropiación y el trato justo y equitativo, bajo los cuales ha sido usual que los Estados se vean compelidos a responder ante demandas de inversores inconformes con intervenciones estatales en escenarios como la salud ${ }^{48}$ o el medio ambiente ${ }^{49}$, donde previamente existían inversionistas en desarrollo de sus actividades ${ }^{50}$.

Toda vez que el contenido de estos estándares no suele estar específicamente definidos en los tratados de inversión, ha correspondido a los tribunales arbitrales definir los términos y condiciones bajo los cuales los Estados pueden invocar su poder regulatorio como argumento válido para limitar el alcance de los derechos garantizados a los inversionistas. Esto es especialmente complejo si se tiene en cuenta que las decisiones en el arbitraje de inversiones no suelen utilizar un sistema de precedente jurisprudencial vinculante, de tal suerte que cada tribunal efectúa su propio análisis con base en las fuentes que considere vinculantes para el caso concreto.

Así las cosas, en términos generales se ha identificado que los árbitros toman en cuenta varios elementos al resolver estas controversias sobre regulación, a saber, la situación particular política y económica específica del Estado o la existencia de conductas que indiquen que el inversor ha contribuido a la violación de los estándares de inversión. Algunos tribunales han tomado en cuenta estos elementos para establecer si existe o no responsabilidad del Estado, y otros apenas como un criterio a tomar en cuenta únicamente en la tasación de la indemnización a cargo del Estado ${ }^{51}$.

Este complejo escenario ha generado que en los últimos años se levanten voces en todos los niveles buscando que se implementen mecanismos que garanticen un mínimo estándar de capacidad regulatoria a los Estados.

En tal sentido la Conferencia de las Naciones Unidas sobre Comercio y Desarrollo en su Informe sobre las Inversiones en el mundo de 2015 indicó que el primero de los principales retos en la reforma de los tratados de inversión es "Salvaguardar el derecho a regular para el logro de los objetivos de desarrollo sostenible. Los All pueden limitar la soberanía de las partes contratantes en la formulación de las políticas nacionales. Por consiguiente, la reforma de los All debe velar por que esos límites no restrinjan indebidamente la formulación legitima de las políticas públicas ni el logro de los objetivos de desarrollo sostenible. Algunas opciones de reforma de los All son aclarar y limitar los estándares de protección de los All (por ejemplo, sobre el trato justo y equitativo, la expropiación indirecta, el trato NMF) y reforzar las "válvulas de escape" (por ejemplo, las excepciones relacionadas con las políticas públicas, la seguridad nacional, las crisis de la balanza de pagos)".

En esta línea, en los años más recientes se han desarrollado tratados de inversión que expresamente reconocen el derecho de los Estados a desarrollar actividades regulatorias en áreas sensibles como el medio ambiente, los derechos laborales, la salud o la seguridad, sea

\footnotetext{
${ }^{47}$ AL FARUQUE (2010), p. 593; SIMMA (2011), p. 580.

${ }^{48}$ Caso Methanex Corp. vs Estados Unidos de América, Myers Inc. vs Canadá, o Técnicas Medioambientales Tecmed S.A. vs México, Philip Morris vs Uruguay, entre otros.

${ }^{49}$ Azurix Corp. Vs Argentina, Compañía de Aguas del Aconquia y Vivendi Universal v. Argentina, entre otros.

${ }^{50}$ CHOUdHURY (2009), p. 990.

51 KNOLL-TUDOR (2009), p. 340
} 
reconociendo expresamente el derecho a regular o insertando cláusulas de excepción donde se salvaguarde tal facultad, como ejemplos de ello se pueden mencionar los tratados bilaterales de inversión celebrados entre Marruecos-Nigeria (artículo 15) ${ }^{52}$, Suiza-México (artículo 3), ChinaCanadá (Artículo 33), Canadá- Eslovaquia (artículo IX) y tratados de Libre comercio como el CETA (artículo 8.9) ${ }^{53}$.

Adicionalmente, se pueden encontrar casos donde el Estado, a partir de un control efectuado por el poder judicial, se ve compelido a renegociar un tratado y/o adoptar declaraciones interpretativas conjuntas para superar defectos o deficiencias respecto a la protección de normas constitucionales. Un ejemplo de esta situación se presentó recientemente en Colombia, donde la Corte Constitucional de este país al efectuar un control de constitucionalidad del tratado bilateral de inversión Francia- Colombia, efectuó varios reparos de constitucionalidad respecto al contenido del tratado, que deberán ser subsanados por el poder ejecutivo buscando la adopción de una declaración interpretativa conjunta con su similar francés ${ }^{54}$.

Si bien estos ejemplos pueden tomarse como indicador de una naciente tendencia dirigida a desarrollar regulaciones más completas, sistemáticas, y garantistas de los Derechos Humanos en los nuevos tratados de inversión, resulta innegable que, respecto al número total de tratados de inversión en vigencia, estos ejemplos resultan ser la excepción y no la regla. Además, es evidente que la renegociación de un tratado o la adopción de declaraciones interpretativas es un proceso lento y complejo cuyo éxito depende de numerosos factores económicos, políticos, sociales y jurídicos.

Sin embargo, el objetivo de lograr tratados de inversión armónicos con las obligaciones de Derechos Humanos no puede ser abandonado por los Estados, por complejo que este sea. En tal sentido, se debe propiciar no solo la inclusión de cláusulas vinculantes -y no meramente voluntarias- de protección y respeto a los Derechos Humanos en el articulado del tratado y su preámbulo, así como buscar que las denominadas cláusulas paraguas desaparezcan de estos tratados o que se utilicen de forma reciproca (no solo a cargo del Estado), propiciar la efectiva inclusión del estándar de debida diligencia al que aluden los denominados Principios Ruggie, establecer parámetros efectivos de prevención y lucha de la corrupción ${ }^{55}$, e incluso acudir al desarrollo de figuras como el principio de manos limpias a fin de asegurar que si existen violaciones de DD.HH por parte del inversor se genere una ausencia de protección de aquel bajo el tratado de inversión ${ }^{56}$. Con acciones de este tipo se podría alcanzar un adecuado punto de intersección entre el derecho de inversiones y los Derechos Humanos.

\section{BIBLIOGRAFÍA CITADA}

Al Faruque, Abdullah (2010): "Mapping the relationship between investment protection and human rights", en: Journal of World Investment Trade (Vol. 11, № 4), pp. 539-560.

BALCERZAK, FILIP (2017): Investor-state arbitration and human rights (Leiden-Boston, Brill/Nijhoff).

BARRAClough, ANDREW y Waincymer, JefF (2005): "Mandatory rules of law in international commercial arbitration", en: Melbourne Journal of International Law (Vol. 6), pp- 205-244.

\footnotetext{
52 Se ha resaltado la técnica innovadora de este tratado, dirigida a lograr un enlace entre la promoción y protección de los derechos del inversionista a la par con los Derechos Humanos, incluyendo tal objetivo desde el mismo preámbulo, pero además desarrollando obligaciones en tal sentido con reglas de carácter vinculante.

53 FEIGHERY (2018), p. 433; BOISSON DE CHAZOURNES (2017), p. 382.

${ }^{54}$ En este caso, uno de los reparos de la Corte Constitucional de Colombia (2019) fue justamente sobre la potencial limitación regulatoria que podría sufrir el Estado colombiano por ciertos contenidos del tratado. Sobre ese particular, señaló esa corporación: “para garantizar el margen de configuración y la autonomía regulatoria del Estado, la Corte estima necesario salvaguardar la competencia de las autoridades públicas para adoptar las medidas que consideren razonables y apropiadas que contribuyan a garantizar el orden público. Esto, en la medida en que las autoridades públicas nacionales, a la luz de la Constitución Política, tienen libertad para determinar el grado de satisfacción del orden público con independencia de la afectación de los inversionistas siempre y cuando no resulte irrazonable el grado de favorecimiento del orden público en comparación con el grado de afectación de los inversionistas".

${ }^{55}$ SHEFFER (2011), pp. 508-516.

${ }^{56}$ KALDUNSKI (2015), p. 69.
} 
Boisson de Chazournes, LaURence (2017): “Environmental Protection and Investment Arbitration: Yin and Yang", en: ACDI Anuario Colombiano de Derecho Internacional (Vol. 10), pp. 371-400.

CHOUdHURY, BARNALI (2009): "Democratic implications arising from the intersection of investment arbitration and human rights", en: Alberta Law Review (Vol. 46, № 4), pp. 983-1008.

COMISIÓN DE DERECHO INTERNACIONAL DE LAS NACIONES UNIDAS (2006): “Documento A/61/10 Informe de la Comisión de Derecho Internacional sobre la labor realizada en su 58 periodo de sesiones, 1 de mayo a 9 de junio y 3 de julio a 11 de agosto de 2006." Disponible en: http://legal.un.org/ilc/publications/yearbooks/spanish/ilc_2006_v2_p2.pdf. [visitado el 15 de agosto de 2019].

Comisión de DeReChos Humanos de LAS NACIONES UnIDAS, SUBComisión de PROMOCIÓN Y PROTECCIÓN (2 de julio de 2003): "Informe del Alto Comisionado para los Derechos Humanos sobre Derechos Económicos, Sociales y Culturales, Los derechos humanos, el comercio y las inversiones, (E/CN.4/Sub.2/2003/9)". Disponible en: https://undocs.org/es/E/CN.4/Sub.2/2003/9. [visitado el 15 de agosto de 2019].

Conferencia de las NaCiones Unidas Sobre Comercio y DeSARRollo CNUCED (2015): "Informe sobre las inversiones en el mundo 2015: Reforma de la gobernanza internacional en materia de inversiones". Disponible en: https://unctad.org/es/PublicationsLibrary/wir2015overview_es.pdf. [visitado el 12 de julio de 2019].

Consejo de Derechos Humanos de las Naciones Unidas (2009). "Informe del Representante Especial del Secretario General de la ONU sobre Derechos Humanos y empresas transnacionales (A/HRC/11/13)". Disponible en: https://www2.ohchr.org/english/bodies/hrcouncil/docs/11session/A.HRC.11.13.pdf. [visitado el 12 de julio de 2019].

Consejo de Derechos humanos de las Naciones Unidas (2014). "Elaboración de un instrumento internacional jurídicamente vinculante sobre las empresas transnacionales y otras empresas con respecto a los derechos humanos (A/HRC/RES/26/9)". Disponible en https://ap.ohchr.org/documents/dpage_e.aspx?si=A/HRC/RES/26/9 [visitado el 15 de julio de 2021].

De BRABANDERE, ERIC (2018): “Human Rights and Foreign Direct Investment", en: Krajewsk, Markus y Hoffmann, Rhea (Eds.), Research Handbook on Foreign Direct Investment. (Cheltenham: Edward Elgar), pp. 619-646.

De Schutter, Oliver; Wouters, Jan; De Man, Philiph y Hachez, Nicolas (2009): "Foreign direct investment, human development and human rights: Framing the issues", en: Human Rights International Legal Discourse (Vol. 3, № 2), pp. 137-176.

DolzeR, RUdolf y SCHREUeR, CHRISTOPH (2012): Principles Of International Investment Law (Oxford, Oxford University Press).

DUMBERRY, PATRICK y DUMAS-AUBIN, GABRIELle (2012): "When and how allegations of human rights violations can be raised in investor-state arbitration", en: Journal of World Investment Trade (Vol. 13, № 3), pp. 349-372.

DUPUY, PIERRE MARIE (2009): “Unification Rather than Fragmentation of International Law? The Case of International Investment Law and Human Rights Law", en: Dupuy, Pierre Marie; Francioni, Francesco y Ulrich, Ernst (Eds.), Human rights in international investment law and arbitration (Oxford, Oxford University Press), pp. 45-63.

FEIGHERY, TIMOTHY J. (2018): "Investor-state arbitration and human rights", en: Vanderbilt Journal of Entertainment Technology Law (Vol. 21, № 2), pp. 417-436. 
FERIA-TINTA, MONICA (2017): "Like Oil and Water? Human Rights in Investment Arbitration in the Wake of Philip Morris v. Uruguay", en: Journal of International Arbitration (Vol 31, № 4), pp. 601 630.

Greenfield, Kent (2018): Corporations are people too (and they should act like it) (New Haven, Londres, Yale University Press).

Hernández Zubizarreta, Juan y Ramiro, Pedro (2015): Contra la lex mercatoria. Propuestas y alternativas para desmantelar el poder de las empresas transnacionales (Barcelona, Icaria).

KALDUNSKI, MARCIN (2015): "Principle of clean hands and protection of human rights in international investment arbitration", en: Polish Review of International and European Law (Vol. 4, № 2), pp. 69-101.

KLEIN, NICOLAS (2012): "Human rights and international investment law: Investment protection as human right", en: Goettingen Journal of International Law (Vol. 4, № 1), pp. 179-196.

KNOLL-TUDOR, IOANA (2009) “The Fair and Equitable Treatment Standard and Human Rights Norms", en: Dupuy, Pierre Marie; Francioni, Francesco y Ulrich, Ernst (Eds.), Human rights in international investment law and arbitration (Oxford, Oxford University Press), pp. 310-344.

KoskenNIEMI, MARTTI (2011): The Politics of International Law (Oxford \& Portland, Hart Publishing). LINARES, ALEJANDRo (2019): El derecho aplicable en el arbitraje de inversión. La tensión con el derecho interno (Bogotá, Universidad Externado de Colombia).

LO, CHANG-FA (2015): "Normative and operational linkages between human rights law and bits building firmer status of human rights in investor-state arbitration", en: Contemporary Asia Arbitration Journal (CAA Journal) (Vol. 8, № 1), pp. 1-28.

HIRSCH, MOSHE (2009): "Investment Tribunals and Human Rights: Divergent Paths", en: Dupuy, Pierre Marie; Francioni, Francesco y Ulrich, Ernst (Eds.), Human rights in international investment law and arbitration (Oxford, Oxford University Press), pp. 97-115.

POLANCO, RODRIGO (s.f): "Arbitraje de inversiones y casos de derechos humanos en América latina". Disponible en: https://law.yale.edu/system/files/area/center/kamel/sela17_polanco_cv_sp.pdf [visitado el 15 de agosto de 2019].

Reiner, Clara y Schreuer, Christoph (2009): "Human Rights and International Investment Arbitration", en: Dupuy, Pierre Marie; Francioni, Francesco y Ulrich, Ernst (Eds.), Human rights in international investment law and arbitration (Oxford, Oxford University Press), pp. 82-97.

SALDIVIA, LAURA (2010): "El derecho y la soberanía en la globalización", en: Teubner, Ghunter; Sassen, Saskia y Krasner, Stephen, Estado, soberanía y globalización (Bogotá, Siglo del Hombre), pp. 19-64.

SCHLIEMANN, CHRISTIAN (2013): "Participation in International Investment Arbitration; A Deconstruction of the Procedural Wall Erected in Joint ICSID Cases ARB/10/25 and ARB/10/15, 12", en; The Law and Practice of International Courts and Tribunals (Vol. 12), pp. 365-390.

SHefFer, Megan (2011): "Bilateral investment treaties: friend or foe to human rights", en: Denver Journal of International Law and Policy (Vol. 39, № 3), pp. 483-522.

SIMMA, BRUNO (2011): "Foreign investment arbitration: place for human rights", en: International and Comparative Law Quarterly (Vol. 60, № 3), pp. 573-596.

SOMMER, CHRISTIAN (2013): “La aplicación de estándares de protección de inversiones extranjeras. Una mirada desde los casos argentinos", en: Anuario Colombiano de derecho Internacional (Vol. 6), pp. 95-130. 
STEINHARDT, RALPH (2005): “Corporate Responsibility and the International law of Human Rights: the new lex mercatoria", en: Alston, Philip (Coord.), Non- State Actors and Human Rights (Oxford, Oxford University Press), pp. 167-182.

SWeetLand, Haley (2016): Shadow Courts: The Tribunals that Rule Global Trade (New York, Columbia Global Reports).

TEUBNER, GUNTHER (1997): "The king's many bodies: the self-deconstruction of law's hierarchy", en: Law and Society Review (Vol. 31, № 4), pp. 763-788.

TEUBNER, GUNTHER (2005): El derecho como sistema autopoiético de la sociedad global (Bogotá, Universidad Externado de Colombia).

TOBAR, JENNER ALONSO (2017): “La autorregulación normativa de los actores privados en el comercio internacional: el caso de los Principios sobre los Contratos Comerciales de UNIDROIT. (Tesis de doctorado, Universidad de Buenos Aires)". Disponible en: http://repositoriouba.sisbi.uba.ar/gsdl/cgibin/library.cgi?a=d\&c=adrposgra\&cl=CL1\&d=HWA_2107 [visitado el 12 de julio de 2019].

VOGEL, DAVID (2009): "The private regulation of global corporate conduct", en; Mattli, Walter y Woods, Ngaire (Eds.), The politics of global regulation (New Jersey, Princeton University Press).

WINKLER, AdAM (2018): We the Corporations: How American Businesses Won Their Civil Rights (New York, W. W. Norton \& Company).

\section{Jurisprudencia citada}

CIADI: BILOUNE VS GHANA (27 de octubre de 1989 y 30 de junio de 1990): Laudos arbitrales Ad Hoc, Lauterpacht, E., Greenwood, C.J. (editores), International Law reports, Vol. 95. Cambridge: Cambridge University Press: pp. 184-232.

UNCITRAL: MYERS INC. VS CANADÁ (30 de diciembre de 2002): laudo arbitral UNCITRAL.

CIADI: TÉCNICAS MEDIOAMBIENTALES TECMED S.A. vs MÉXICo (29 de mayo de 2003): laudo arbitral CIADI. Caso número ARB (AF)/00/2.

UNCITRAL: Methanex CoRP. vs Estados Unidos de AmÉRICA (3 de agosto de 2005): laudo arbitral UNCITRAL.

CIADI: AZURIX CoRp. vs ARgentina (14 de julio de 2006): laudo arbitral CIADI. Caso número ARB/01/12.

CIADI: WORLD DUTY FREE Co LTD VS KenYA (4 de octubre de 2006): laudo arbitral CIADI. Caso número $\mathrm{ARB} / 00 / 7$.

CIADI: Channel Tunnel Group vs Reino Unido y Francia (30 de enero de 2007): laudo parcial. Disponible en: https://www.jstor.org/stable/20488241.

CIADI: United PARCel SeRvices of America INC. vs CANAdÁ (24 de mayo de 2007): laudo arbitral UNCITRAL. Caso número UNCT/02/1.

CIADI: CompañIA DE AgUAS DEL ACONQUIJAS. A. Y VIVENDI UniVERSAL S. A. vS ARGENTINA (20 de agosto de 2007): laudo arbitral CIADI. Caso número ARB/97/3.

CONSEJO DE DEREChOS Humanos de LAS NACIONES UnidAS (7 de abril de 2008), Resolución A/HRC/8/5.

CIADI: GLAMIS GOLD, LTD VS ESTADOS UNIDOS (8 de junio de 2009): Laudo arbitral UNCITRAL.

CIADI: Quasar de Valores SICAV S.A. y otros vs Federación de Rusia (20 de julio de 2012): laudo arbitral de la Cámara de Comercio de Estocolmo. Caso número 24 de 2007. 


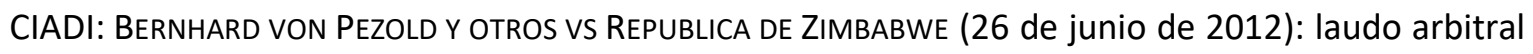
CIADI. Caso número ARB/10/15. Orden procesal № 2, Casos NO. ARB/10/15 y ARB/10/25.

CIADI: RoMPETRol GRoup N.V. vs RUMANI (6 de mayo de 2013): laudo arbitral CIADI. Caso número $\mathrm{ARB} / 06 / 3$.

Colombia: Sentencia T-197 de 2014 (Sentencia del 1 de abril de 2014), Corte Constitucional de la República de Colombia, M.P: Alberto Rojas Ríos.

Consejo de Derechos humanos de las Naciones Unidas (25 de junio de 2014), Resolución A/HRC/26/L.22.

UNCITRAL: HeSham T. M. Al WARRAQ vs INDONESIA (15 de diciembre de 2014): laudo arbitral UNCITRAL.

CIADI: Tulip Real Estate And DeVelopment Netherlands B.V vs Turquia (30 de diciembre de 2015): Decisión de anulación laudo arbitral CIADI. Caso número ARB/11/28.

CIADI: HOUBEN VS BURUNDI (12 de junio de 2016): Laudo arbitral CIADI. Caso número ARB/13/7.

CIADI: PhILIP Morris Brands SÀrl, Philip Morris Products S.A.y Abal Hermanos S.A. vs URUguay (8 de julio de 2016): laudo arbitral CIADI. Caso número ARB/010/07.

CIADI: URBASER S.A. Y CONSORCIO DE AGUAS BILBAO BIZKAIA, BILBAO BISKAIA UR PARTZUERGOA VS ARGENTINA (8 de diciembre de 2016): laudo arbitral CIADI. Caso número ARB/07/26.

Colombia: Sentencia C-252 de 2019 (6 de junio de 2019), Corte Constitucional de la República de Colombia, M.P: Carlos Bernal Pulido. 